\title{
Cornering diphoton resonance models at the LHC
}

\section{Mihailo Backović, ${ }^{a}$ Suchita Kulkarni, ${ }^{b}$ Alberto Mariotti, ${ }^{c}$ Enrico Maria Sessolo ${ }^{d}$ and Michael Spannowsky ${ }^{e}$}

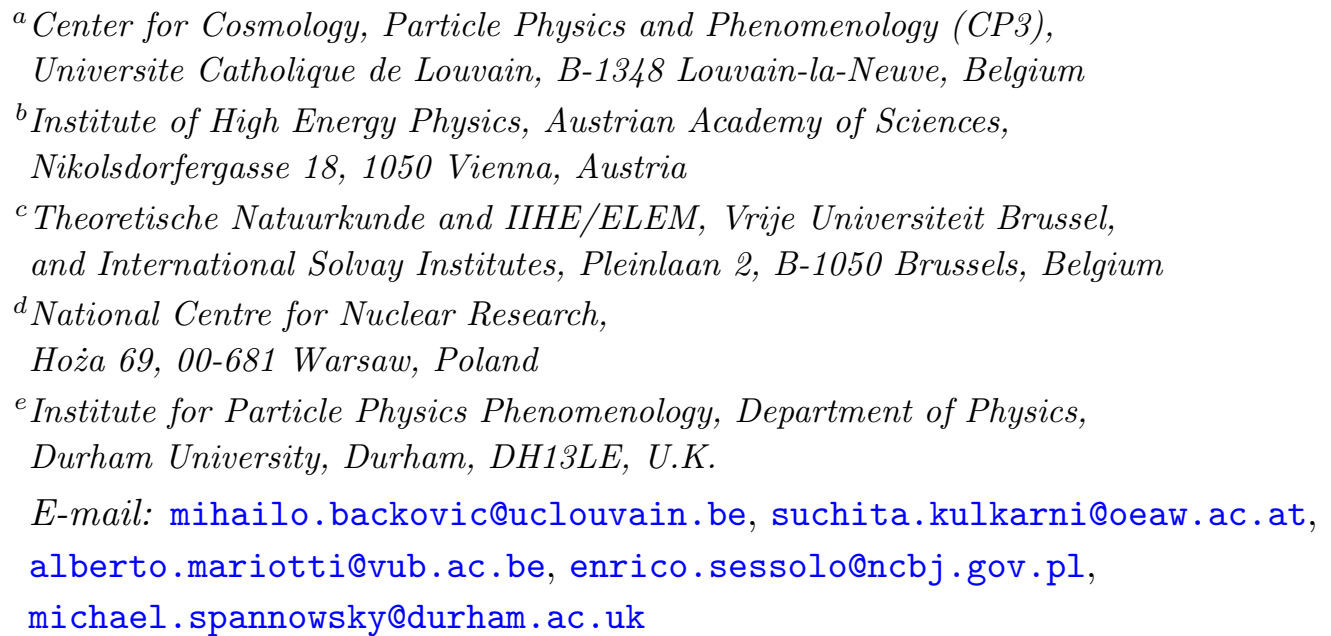

ABSTRACT: We explore the ability of the high luminosity LHC to test models which can explain the $750 \mathrm{GeV}$ diphoton excess. We focus on a wide class of models where a $750 \mathrm{GeV}$ singlet scalar couples to Standard Model gauge bosons and quarks, as well as dark matter. Including both gluon and photon fusion production mechanisms, we show that LHC searches in channels correlated with the diphoton signal will be able to probe wide classes of diphoton models with $\mathcal{L} \sim 3000 \mathrm{fb}^{-1}$ of data. Furthermore, models in which the scalar is a portal to the dark sector can be cornered with as little as $\mathcal{L} \sim 30 \mathrm{fb}^{-1}$.

Keywords: Phenomenological Models

ARXiv EPrint: 1605.07962 


\section{Contents}

1 Introduction 1

2 General strategy and LHC limits 2

3 Diphoton resonance models $\quad 5$

3.1 The "vanilla" model: $g_{X}=g_{f}=0 \quad 6$

$\begin{array}{ll}3.2 \text { The dark matter model: } g_{X} \neq 0, g_{f}=0 & 10\end{array}$

3.3 The "top-philic" model: $g_{X}=0, g_{f} \neq 0 \quad 11$

$\begin{array}{lll}4 & \text { Summary } & 13\end{array}$

$\begin{array}{ll}\text { A Limit extrapolation } & 14\end{array}$

$\begin{array}{ll}\text { B Analytical form of the decay widths } & 17\end{array}$

\section{Introduction}

Both ATLAS and CMS collaborations recently announced an excess in the diphoton spectrum around the invariant mass of $m_{\gamma \gamma} \approx 750 \mathrm{GeV}$. While the excess is not statistically significant to claim a discovery (ATLAS finds a local significance of $3.6 \sigma[1,2]$ and CMS one of $3.0 \sigma[3,4])$, it is certainly interesting to entertain the idea that the data points to the existence of a new particle.

If such particle is a singlet under the SM gauge group, it is inevitable that the diphoton excess will be correlated with signals in other channels involving gauge bosons (e.g. $Z \gamma$, $Z Z$, or $W W$ ). It has been shown that an excess should appear at least in one of the before-mentioned channels, regardless of the underlying model parameters $[5,6]$. In the optimistic scenario where the $750 \mathrm{GeV}$ diphoton excess remains as more data comes in, measurements of other final states which are correlated to the diphoton excess will hence become instrumental in both confirming the signal, as well as determining the properties of the new particle. In particular, not observing correlated signals in final states with Standard Model (SM) gauge bosons will have direct implications on many scenarios attempting to explain the excess.

The width of the diphoton excess offers additional crucial information about the nature of the possible new particle. The line-shape of the excess measured by ATLAS indicates a rather broad resonance with a width $\Gamma_{\text {tot }} \simeq 45 \mathrm{GeV}$, which is difficult to account for if it decays only into Standard Model (SM) particles. Large unobserved decay modes can point to interactions between the new resonance and dark matter, leading to collider signatures in channels with large missing energy, as well as signals in direct dark matter detection experiments via scattering off nuclei, and the measurements of galactic $\gamma$-ray fluxes [7-11]. 
In this paper we explore the reach of LHC run-2 searches for the diphoton resonance models. Leading order approximations for the production of $s$-channel resonances allow for the use of simple scaling rules to study the constraints of existing and future LHC results on the model parameter space. Specifying the features for the diphoton excess, such as the production mechanism and cross section, defines a hyper-surface in the multi-dimensional parameter space which can explain the excess. Our approach consists in constraining these surfaces further, by imposing collider bounds on correlated final states.

Using concrete examples, we demonstrate the most sensitive channels and relevant bounds, as well as the required integrated luminosity to rule out particular models explaining the diphoton excess. For concreteness, we assume throughout the paper that the resonance is a scalar singlet under the SM gauge group. Hence, its interactions with SM particles are captured at leading order by a set of dimension- 5 operators suppressed by a new physics scale $\Lambda$ [12]. We further assume that the new resonance does not mix with the SM Higgs boson, as existing and projected limits from Higgs coupling measurements set strong indirect constraints [13, 14].

We discuss three concrete benchmark scenarios, which serve to encompass a large class of $750 \mathrm{GeV}$ diphoton resonance models. First, we study the "vanilla" scenario, in which a scalar singlet couples only to SM gauge bosons via dimension-5 effective interactions. Second, we consider a scenario in which decays of a $750 \mathrm{GeV}$ scalar into an invisible sector (i.e. dark matter) accommodate the potentially large resonance width. Finally, we analyze a scenario in which the scalar is allowed to couple to SM quarks in addition to SM gauge bosons. For the purpose of studying future LHC limits on the three scenarios, we project existing 8 and $13 \mathrm{TeV}$ limits on production of gauge boson, mono-jet, and $t \bar{t}$ final states at various luminosities. We outline the strategy we adopt and the simplified approach we employ to project limits for the LHC in section 2. In section 3 we present our main results, where we confront concrete diphoton scenarios with the existing LHC bounds and our estimated projections for the $13 \mathrm{TeV}$ run. Finally, we briefly summarize our results and conclude in section 4. In appendix A we provide more technical details about limit projection and in appendix B we review the analytical forms used here for the calculation of the decay widths.

\section{General strategy and LHC limits}

We begin with a brief discussion of the possible production modes for the $750 \mathrm{GeV}$ diphoton resonance. We limit our discussion to the case of a pure scalar, however most of the qualitative conclusions in our paper will hold in the case of a pseudo-scalar resonance as well. In the most general scenario, the onshell production cross section of the scalar resonance can be approximated by

$$
\sigma(p p \rightarrow S) \approx \sum_{i j} C_{i j}(s, M) \sigma_{i j}
$$

where $i, j$ are proton constituents (including photons), $C_{i j}$ are the dimensionless parton luminosity factors and $\sigma_{i j}$ are partonic cross sections. Limits from $8 \mathrm{TeV} \mathrm{LHC}$ disfavor 
production via light quarks $[12,15]$ and we will hence limit ourselves to scenarios in which the new scalar particle is produced via either gluon fusion $(g g)$ or photon fusion $(\gamma \gamma)$ initial state.

The photon fusion production mechanism deserves further discussion. Production of a scalar resonance compatible with the diphoton excess via photon fusion are studied in refs. [16-20]. ${ }^{1}$ However, it is important to note that many subtleties arise in considering the photon fusion channel. The cross section enhancement between 8 and $13 \mathrm{TeV}$ centerof-mass energy at the LHC is subject to large uncertainties and can vary between a factor 2 and $4[17,18]$. Hence, pure photon production is possibly already in tension with $8 \mathrm{TeV}$ data if the ratio is closer to 2 . In addition, given the inclusive nature of the diphoton excess measurements in the ATLAS and CMS searches, it is also possible that vector boson fusion (VBF) channels with one or two additional reconstructed jets contribute to the overall production cross section. We estimated the VFB contributions with one or two additional jets for the models we consider in this paper. We found that VBF contributes at most $\sim 15 \%$ of the inclusive diphoton production cross section in the regions of the parameter space compatible with the observed diphoton excess. ${ }^{2}$ We will thus neglect such VBF contributions in the following.

Continuing, within the narrow width approximation the diphoton cross section at leading order is simply

$$
\begin{aligned}
\sigma_{\gamma \gamma} & =\left[\sigma_{\gamma}(p p \rightarrow S)+\sigma_{g}(p p \rightarrow S)\right] \times \operatorname{Br}(S \rightarrow \gamma \gamma) \\
& =\left[c_{\gamma}^{2} \sigma_{\gamma}(p p \rightarrow S)_{c_{\gamma}=1}+c_{G}^{2} \sigma_{g}(p p \rightarrow S)_{c_{G}=1}\right] \times \operatorname{Br}(S \rightarrow \gamma \gamma),
\end{aligned}
$$

where we have factored out the dependence on $S$ couplings to gluons and photons $\left(c_{G}\right.$ and $\left.c_{\gamma}\right) . \sigma_{\gamma, g}$ are the photon and gluon initiated production cross sections respectively. Note that $\operatorname{Br}(S \rightarrow \gamma \gamma)$ is an implicit function of all of the theory parameters. Assuming a signal cross section $\sigma_{\gamma \gamma}^{*}$, consistent with the observed excess, eq. (2.1) can be solved for $c_{G}^{*}$ as a function of the remaining parameters in a given model, hence defining a slice of the parameter space which can accommodate the excess. Note that in the limit of $c_{\gamma} \rightarrow 0$ the branching ratio into photons also vanishes, yielding no viable solution for $c_{G}^{*}$.

Parameter space slices determined by $\sigma_{\gamma \gamma}^{*}$ can then be bound by searches in the complementary final state channels. ATLAS and CMS have recently published the first results from the LHC $13 \mathrm{TeV}$ run, with an integrated luminosity of $3.2 \mathrm{fb}^{-1}$ and $2.3 \mathrm{fb}^{-1}$ respectively, which can be used to constrain existing models. Bounds from resonance searches involving gauge bosons final states are of particular relevance for constraining gauge invariant parameterizations of the diphoton models.

We present a summary of the bounds used in this paper in table 1. In the $Z \gamma$ final state the $95 \%$ C.L. $8 \mathrm{TeV}$ ATLAS upper bound on the production cross section times branching ratio [23] reads approximately $11 \mathrm{fb}$, whereas the bound from the equivalent search in Run 2 [24] yields $\sim 30 \mathrm{fb}$. While the data from Run 2 is not particularly useful to constrain these scenarios yet, it can nonetheless be used to estimate the reach of these searches for

\footnotetext{
${ }^{1}$ First coupling constraints for such models using $8 \mathrm{TeV}$ data have been obtained in [21].

${ }^{2}$ The full treatment of multi-jet merging in electroweak processes is beyond the scope of our paper [22].
} 


\begin{tabular}{|ccccccc|}
\hline Search & $\begin{array}{c}8 \mathrm{TeV} \text { limit }[\mathrm{fb}] \\
\text { (observed) }\end{array}$ & $\begin{array}{c}13 \mathrm{TeV} \text { limit }[\mathrm{fb}] \\
\text { (observed) }\end{array}$ & $\mathcal{L}=3.2 \mathrm{fb}^{-1}$ & $\mathcal{L}=30 \mathrm{fb}^{-1}$ & $\mathcal{L}=300 \mathrm{fb}^{-1}$ & $\mathcal{L}=3000 \mathrm{fb}^{-1}$ \\
\hline$Z \gamma$ & $11[23]$ & $30[24]$ & 43 & 14 & 4.4 & 1.4 \\
$Z Z$ & $12[25]$ & $180[26]$ & 82 & 27 & 8.5 & 2.7 \\
$W W$ & $40[27]$ & $400[28]$ & 300 & 98 & 31 & 9.8 \\
$t \bar{t}$ & $460[29]$ & $10000[30]$ & 3267 & 1067 & 337 & 107 \\
$\mathrm{MET}+j$ & 7.2 (SR7) [31] & 61 (IM5) [32] & 51 & & & 0.5 \\
& & 19 (IM7) [32] & 15 & 5 & 1.5 & 0.5 \\
\hline
\end{tabular}

Table 1. Extrapolations of experimental limits relevant for the $750 \mathrm{GeV}$ diphoton. The models are constrained by the strongest of the $8 \mathrm{TeV}$ and $13 \mathrm{TeV}$ observed limits. The inclusive regions SR7 (for the mono-jet $(\mathrm{MET}+j) 8 \mathrm{TeV}$ search) and IM5 (for the corresponding $13 \mathrm{TeV}$ results) are charecterized by $E_{T}^{\text {miss }}>500 \mathrm{GeV}$. The inclusive region IM7 for the $13 \mathrm{TeV}$ search is defined by $E_{T}^{\text {miss }}>700 \mathrm{GeV}$. For the $Z Z$ and $t \bar{t}$ searches the expected limit at $3.2 \mathrm{fb}^{-1}$ is extrapolated from the $8 \mathrm{TeV}$ expected bound (see text).

future luminosity. The idea is based on the assumption that, while being model dependent, quantities like cross sections, acceptances and efficiencies do not depend on the integrated luminosity. In the limit of a large number of events, one can obtain the expected 95\% C.L. cross section bound at any target luminosity $\mathcal{L}$ by rescaling the $3.2 \mathrm{fb}^{-1}$ limit with the ratio of the square root of corresponding luminosities. Considering, for example, the $Z \gamma$ case in table 1 , rescaling the expected $3.2 \mathrm{fb}^{-1}$ bound of $\sim 43 \mathrm{fb}[24]$ yields the projected values shown in the columns of 30,300 , and $3000 \mathrm{fb}^{-1}$. $^{3}$

We use the above luminosity-rescaling ansatz to obtain the majority of the projections considered in this paper. However, while luminosity rescaling provides conservative estimates in most cases, it does not always reproduce the most realistic expectations. As experience with the large number of search results produced during and after the $8 \mathrm{TeV}$ run has shown, a statistical combination of the data obtained in searches sensitive to different final states often leads to a dramatic improvement in the bounds with respect to searches in single channels. For instance, a direct comparison of the expected $8 \mathrm{TeV}$ bounds on the production cross section of a heavy scalar decaying to $Z Z$ in the $l l l l, l l(\nu \nu) q q, l l \nu \nu$, and a combination thereof [25] shows that the combined limit is at least a factor of two stronger than any of the individual bounds. ATLAS has published results for the $13 \mathrm{TeV}$ $Z Z$ resonance searches in the $\nu \nu q q[35], l l q q[36]$, and $l l \nu \nu$ [26] final states, but at this early stage the combination has not been published. It is reasonable to assume that the final combined limit will be also stronger than the one obtained in refs. [35, 36], or [26]. Hence, we will adopt the $13 \mathrm{TeV} Z Z$ limit extrapolated from the combined $8 \mathrm{TeV}$ LHC limit, using the procedure described in detail in appendix $A$. We have verified that the procedure accurately reproduces the existing $13 \mathrm{TeV}$ limits in the $l l q q$ and $\nu \nu q q$ channels, leading us to conclude that our combined limit extrapolation is also accurate (see appendix A for more details).

\footnotetext{
${ }^{3}$ We stress that the limits we obtain in this way are conservative. Data-driven methods can reduce systematic uncertainties when large data samples are available and dedicated reconstruction techniques [33, 34]. exploiting the increased center-of-mass energy at $13 / 14 \mathrm{TeV}$ and different decay mode scan improve on the limits we extrapolate.
} 
Limits on the resonant $W W$ production both at $8 \mathrm{TeV}$ and $13 \mathrm{TeV}$ exist $[27,28]$, and we adopt the observed limits on the $750 \mathrm{GeV}$ resonance from both LHC runs.

The strongest observed ATLAS limits in the final state with at least one jet and large missing transverse momentum $E_{T}^{\text {miss }}$ (hereafter $\mathrm{MET}+j$ ) comes from inclusive search bins denominated SR7 (in the $8 \mathrm{TeV}$ search [31]) and IM5 (at $13 \mathrm{TeV}$ [32]), which are defined by $E_{T}^{\text {miss }}>500 \mathrm{GeV}$. The strongest expected limit at $13 \mathrm{TeV}$ comes instead from the inclusive bin IM7 with $E_{T}^{\text {miss }}>700 \mathrm{GeV}$. Hence, for the purpose of extrapolating the limit to higher luminosities we use the expected limit at $13 \mathrm{TeV}$ in the inclusive bin IM7.

Finally, current experimental searches for $t \bar{t}$ resonances at $13 \mathrm{TeV}$ [30] have focused only on the boosted regime, with no publicly available result on searches for $t \bar{t}$ resonances in the resolved regime. Boosted top analyses are ill suited for efficient reconstruction of the $t \bar{t}$ final states with invariant mass of $\lesssim 1 \mathrm{TeV}$ (assuming the standard fat jet cone of radius $R=1.0$ ), resulting in $13 \mathrm{TeV}$ limits on a $750 \mathrm{GeV}$ resonance which are far weaker than the extrapolated $8 \mathrm{TeV}$ limits in the resolved jet analysis. For $13 \mathrm{TeV} t \bar{t}$ final state, we hence adopt an extrapolated limit from the resolved $8 \mathrm{TeV}$ analysis, obtained with the techniques explained in appendix A.

\section{Diphoton resonance models}

In order to illustrate the strategy we have discussed in the previous section, we consider a concrete set of models where the new resonance is represented by a singlet scalar coupled to the SM with dimension-five operators. Moreover, we also investigate the possibility that the new resonance plays the role of a portal to a dark sector. A wide class of diphoton resonance models can comprehensively be described by the interaction Lagrangian

$$
\mathcal{L} \supset \frac{c_{G}}{\Lambda} S G^{\mu \nu} G_{\mu \nu}+\frac{c_{W}}{\Lambda} S W^{\mu \nu} W_{\mu \nu}+\frac{c_{B}}{\Lambda} S B^{\mu \nu} B_{\mu \nu}+g_{f} \sum_{q} \frac{m_{q}}{\Lambda} S \bar{q} q+g_{X} S \bar{X} X
$$

where $G_{\mu \nu}, W_{\mu \nu}$, and $B_{\mu \nu}$ are the $\mathrm{SU}(3), \mathrm{SU}(2)$, and U(1) field strength tensors, respectively, $q$ indicates SM fermions (of mass $m_{q}$ ), and $X$ is an invisible Dirac fermion which can play the role of dark matter. In the following we will independently study different subsets of this general class of models by switching on and off some of the couplings in eq. (3.1).

Note that we assumed that the new scalar resonance does not couple to the SM Higgs boson. The coupling to the Higgs is mainly constrained by the allowed size of the mixing angle, which is bounded by LHC Higgs coupling measurements to be $\lesssim O(10-20 \%)$ [13]. This already puts significant constraints on possible correlated signals of the new resonance in the Higgs final states, and we leave to future studies a detailed investigation of the LHC $13 \mathrm{TeV}$ reach for these signatures.

We point out that the couplings of the scalar are chosen proportional to the quark masses, to respect minimal flavor violation. Since $S$ is a singlet of the SM gauge groups, the new couplings to SM fermions should be considered as descending from dimensionfive operators such as $\frac{1}{\Lambda} y S H \bar{Q}_{L} u_{R}$, which after electroweak symmetry breaking, generate the couplings in eq. (3.1). The couplings with SM fermions in eq. (3.1) have an extra suppression factor scaling, $m_{q} / \Lambda$, for this reason. Without loss of generality, we have 
introduced a unique suppression scale $\Lambda$ for the various operators, which are then weighted by different $O(1)$ couplings $\left(c_{G}, c_{W}, c_{B}, g_{f}\right)$. For definiteness we will take $\Lambda=10 \mathrm{TeV}$ throughout the paper.

As mentioned before, we will consider a combination of production mechanisms. For couplings of similar size gluon-fusion is typically the dominant production mechanism. However we will explore also regions of the parameter space where photon-fusion processes, which scale like $c_{\gamma}^{2} \equiv\left(c_{B} \cos ^{2} \theta_{W}+c_{W} \sin ^{2} \theta_{W}\right)^{2}$, are dominating. In the case of quarkinitiated production, the dimensionless Yukawa couplings of $S$ to the quarks are suppressed by a factor $g_{f} m_{q} / \Lambda$, as they descend from higher dimensional gauge invariant operators. Hence, the light quark contributions are suppressed by the small quark masses, while the heavy quark ones are suppressed by small proton PDF and by the smallness of $g_{f} m_{q} / \Lambda$ (since we are considering $O(1)$ couplings and $\Lambda \gg m_{q}$ ). In particular, the top loop induced gluon fusion contribution to the $S$ production cross section is negligible with respect to other production mechanisms in the range of couplings that we study.

In order to estimate the production cross section for the resonance $S$ through the available processes we make use of several tools. We have implemented the model of eq. (3.1) in FeynRules [37] and we simulate the production of $S$ at the LHC using MadGraph5_aMC@NLO (MG5_aMC) [38] with the NN23LO1 [39] PDF set for gluon as well as for photon PDFs. For photon-fusion we consider both the inelastic-inelastic as well as the elastic-inelastic proton scattering processes.

Given the production cross section for the resonance, the cross sections in the various final states are determined by the branching ratios. Analytic formulas for the partial decay widths of $S$ in the model (3.1) are listed in appendix B.

In exploring the parameter space of the model, our strategy relies on solving the condition $\sigma_{\gamma \gamma}=\sigma_{\gamma \gamma}^{*}$ (see eq. (2.1)) for the coupling $c_{G}$. After fixing the couplings $g_{X}$ and $g_{f}$ to some representative value, we present the results in the $\left(c_{B}, c_{W}\right)$ plane. For definiteness we choose $\sigma_{\gamma \gamma}^{*}=7 \mathrm{fb}$ but our results are qualitatively robust under change of the required cross section. We will also display the $c_{G}$ contours necessary to fit the excess, and identify the most relevant production mechanism on each region of the parameter space.

\subsection{The "vanilla" model: $g_{X}=g_{f}=0$}

We start our analysis by considering the simplest version of the model capable of explaining the diphoton excess, i.e. we set the couplings to dark matter and SM fermions to 0. The so called "vanilla" model is then parameterized only by three couplings: $c_{W}, c_{B}$ and $c_{G}$. We explore the parameter space in the range $\left(c_{B}, c_{W}\right) \in\{-1,1\}$ and for every value of $\left(c_{B}, c_{W}\right)$ we solve the equation $\sigma_{\gamma \gamma}=\sigma_{\gamma \gamma}^{*}=7 \mathrm{fb}$ for $c_{G}^{2}$, imposing the conservative bound $c_{G}<4 \pi$.

Figure 1 shows our first result. In the upper left plot of figure 1 we display in solid red the contours of $c_{G}$ consistent with signal cross section $\sigma_{\gamma \gamma}^{*}$. The values of $c_{G}$ decrease towards larger values of $c_{B}$ and $c_{W}$ since the branching ratio into photons increases. In addition, the photon fusion contribution to the total production cross section also increases with larger $c_{B}$ and $c_{W}$ values, requiring a lower gluon fusion contribution to reproduce the signal. 

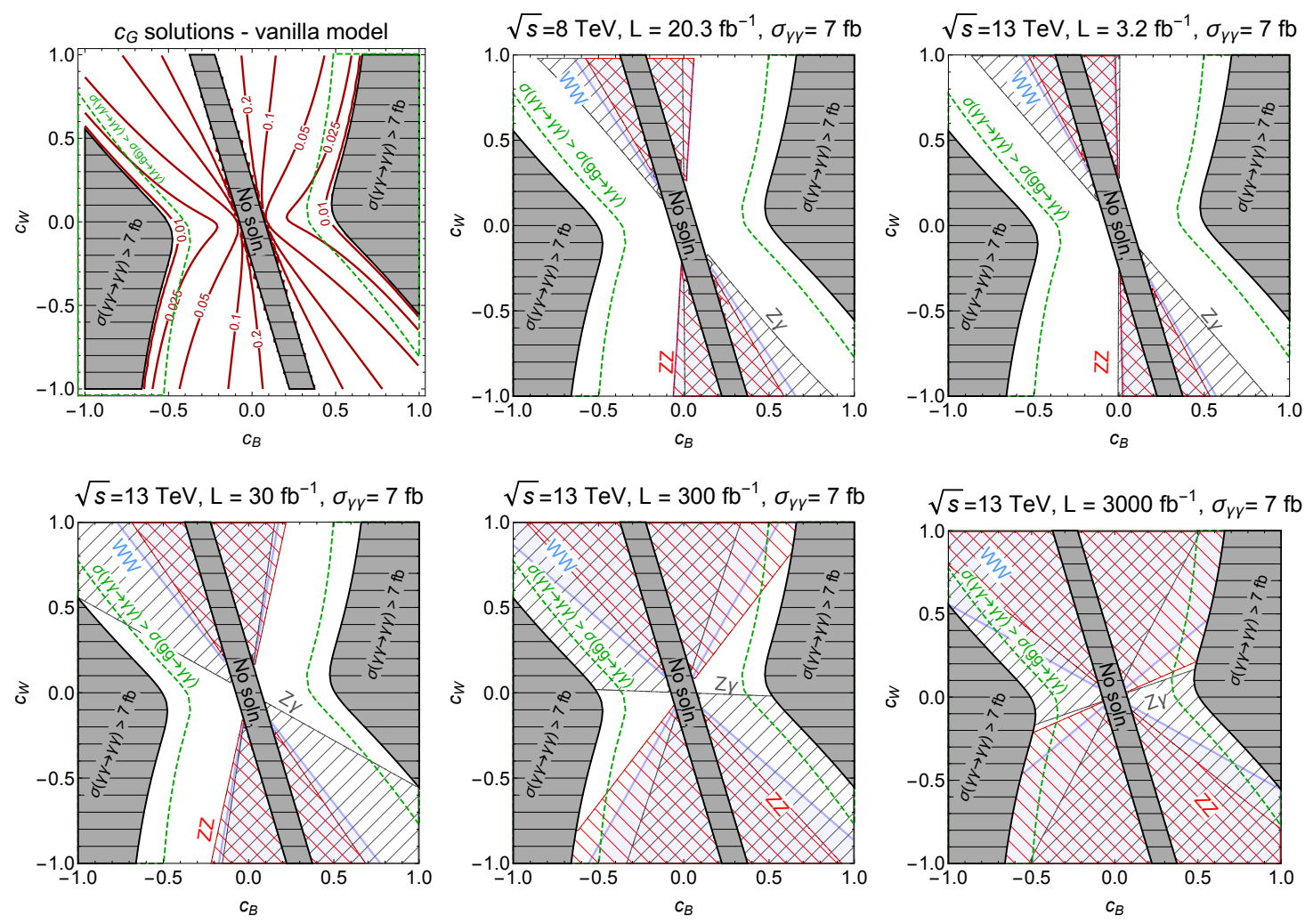

Figure 1. Analysis of the $\left(c_{B}, c_{W}\right)$ plane in the vanilla model parameter space. We apply the bounds from the 8 and $13 \mathrm{TeV}$ runs of the $\mathrm{LHC}$ as well as present several projections in future luminosities. The dark grey shaded regions are where there we find no $c_{G}$ solution for the diphoton excess. Other shaded regions are excluded by particular searches labeled on the plots. The upper left panel shows the contours of constant $c_{G}$, necessary to accommodate the required signal cross section.

The green dashed contours mark the boundary between regions where gluon fusion dominates and regions where photon fusion dominates instead. Photon fusion can be dominant only for large values of $c_{B}$. The shape of the green dashed contour is determined by the competition between the $\operatorname{BR}(S \rightarrow \gamma \gamma)$ and the branching ratios for the other electroweak bosons $(Z \gamma, Z Z$, and $W W)$, which can deplete the signal in $\gamma \gamma$.

The gray regions indicate regions where no solutions for $c_{G}$ resulting into $\sigma_{\gamma \gamma}=\sigma_{\gamma \gamma}^{*}$ exist. We can identify two distinct gray areas which have different physical interpretations. The almost vertical gray stripe close to the central axis (denoted "No-soln.") is located around the straight line $c_{\gamma}=0$. In this regime, the coupling to photons is very small, leading to the fact that no real value of $c_{G}$ can reproduce the signal strength $\sigma_{\gamma \gamma}^{*}$. The argument can be understood analytically as follows. The coupling to photons is almost vanishing in the central grey region, leading to a gluon fusion dominated production mechanism. We can then write

$$
\sigma_{g g}(p p \rightarrow S \rightarrow \gamma \gamma)=\frac{\mathcal{C}_{g g}}{m_{S} s} \frac{\Gamma_{g g} \Gamma_{\gamma \gamma}}{\Gamma_{\text {tot }}}
$$

where $\mathcal{C}_{g g}$ is the gluon luminosity and $s$ is the centre of mass energy. One can impose 
$\sigma_{g g}(p p \rightarrow S \rightarrow \gamma \gamma)=\sigma_{\gamma \gamma}^{*}$ and solve this equation for the total width of $S$ obtaining

$$
\Gamma_{\text {tot }}=\frac{\mathcal{C}_{g g}}{\sigma_{\gamma \gamma}^{*} m_{S} s} \Gamma_{g g} \Gamma_{\gamma \gamma}
$$

Given that the total width of the resonance is always larger or equal than the width into gluons, $\Gamma_{\text {tot }} \geq \Gamma_{g g}$, we arrive to the inequality

$$
\Gamma_{\gamma \gamma} \geq \frac{\sigma_{\gamma \gamma}^{*} m_{S} s}{\mathcal{C}_{g g}},
$$

which implies an absolute lower bound for the partial decay width into photons, necessary to accommodate the diphoton excess. Inserting the explicit expression for the $\gamma \gamma$ partial width (see appendix B) we obtain the lines which delimit the vertical gray stripe:

$$
c_{W}=-c_{B} \tan ^{-2} \theta_{W} \pm \frac{2 \sqrt{\pi s \sigma^{*}} \Lambda}{m_{S} \sqrt{\mathcal{C}_{g g}} \sin ^{2} \theta_{W}} .
$$

The above argument does not depend on the other contributions to $\Gamma_{\text {tot }}$ and is therefore a generic result for the complete model of eq. (3.1), independently of the value of $g_{X}$ and $g_{f}$. We will indeed find the same gray stripe around $c_{\gamma}=0$ in all of the other scenarios considered in this paper.

The other gray region, denoted with $\sigma_{\gamma \gamma}>7 \mathrm{fb}$ in figure 1 , are instead characterized by excessively large rates in $\gamma \gamma$, completely dominated by photon-fusion processes. The internal border of the region identifies the line where the production mechanism is $100 \%$ photon fusion, and $c_{G}=0$.

Figure 1 does not show the $8 \mathrm{TeV}$ bound on $\gamma \gamma$ final states. In the region where gluon fusion dominates, this bound is automatically satisfied since gluon luminosity increases by a factor of 4.7 , and hence a $\sigma_{\gamma \gamma}(13 \mathrm{TeV})=7 \mathrm{fb}$ corresponds to $\sigma_{\gamma \gamma}(8 \mathrm{TeV})=1.49 \mathrm{fb}$, just below the LHC $8 \mathrm{TeV}$ bound. In the photon-fusion dominated regions the argument is less straightforward. Using the NN23LO1 PDF in MG5 the enhancement factor from 8 to $13 \mathrm{TeV}$ in photon fusion is approximately 2 and hence the photon-fusion dominated regions would not be compatible with LHC $8 \mathrm{TeV}$ constraints. Given the on-going discussion in the literature about the exact value of the enhancement factor [18], it is still possible that photon-fusion is eventually a viable option [16-19]. Thus, given the large uncertainties in such estimate, conservatively we do not impose any extra bound on such regions from the LHC $8 \mathrm{TeV} \gamma \gamma$ final state searches. An ATLAS study of the jet multiplicity distribution in the diphoton events seems to show that the data favors production processes with a small number of accompanying jets [2], hence consistent with dominant photon-fusion. For all of the above reasons, we choose to simply denote the region with a dashed green line, and remain agnostic on whether it is viable or not.

We proceed to investigate the bounds which are imposed by the LHC $8 \mathrm{TeV}$ searches of resonances in the $Z Z, Z \gamma$, and $W W$ final states. The results for the $8 \mathrm{TeV}$ limits are displayed in the second top panel of figure 1, where as usual on every point of the plane we have solved for $c_{G}$ in order to get $\sigma_{\gamma \gamma}(13 \mathrm{TeV})=7 \mathrm{fb}$. The signal cross section in electroweak boson final states, once the signal yield in $\gamma \gamma$ is imposed, is only a function of 

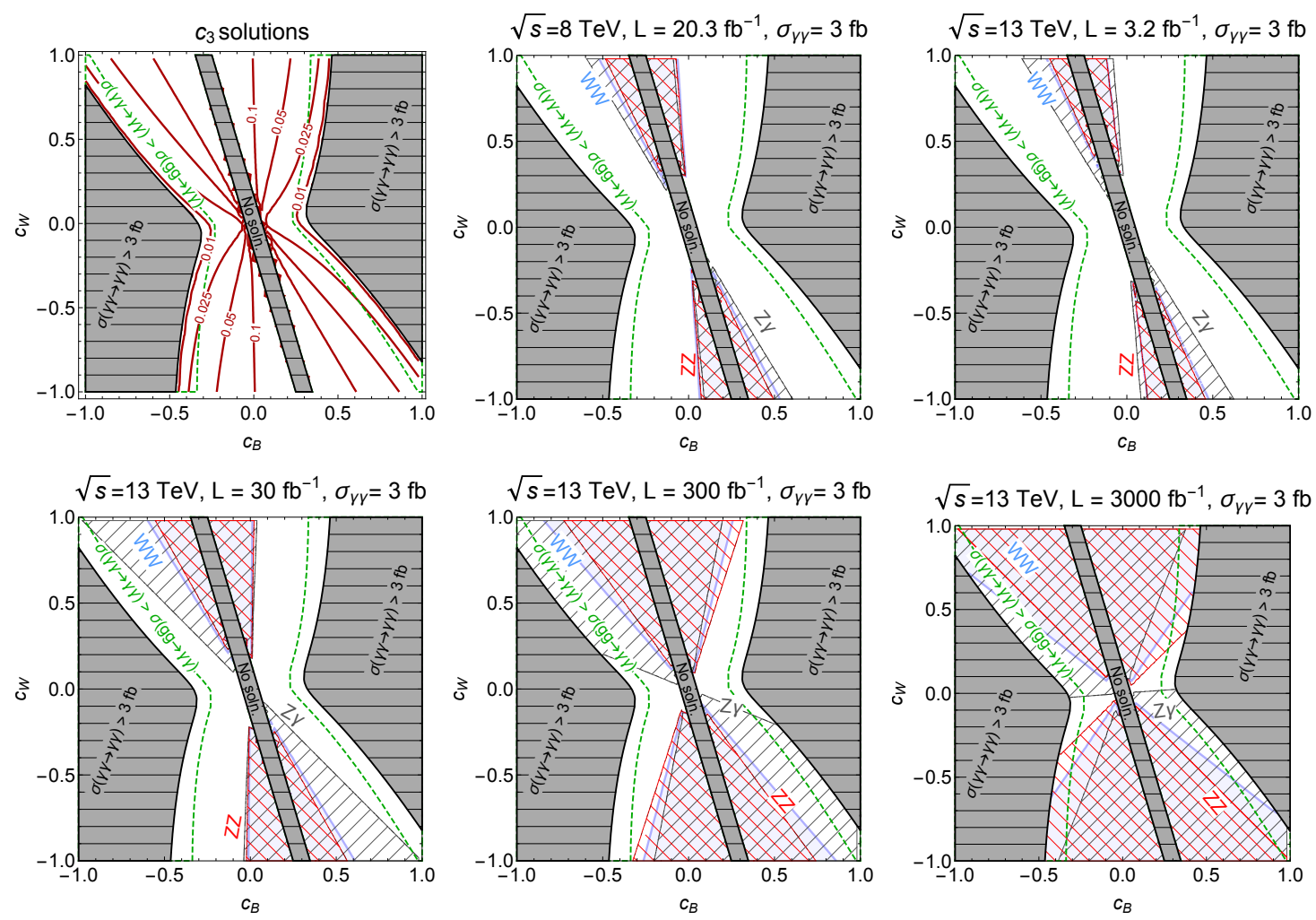

Figure 2. Analysis of the $\left(c_{B}, c_{W}\right)$ plane in the vanilla model parameter space for $\sigma_{\gamma \gamma}^{*}=3 \mathrm{fb}$. As in figure 1, we apply the bounds from the 8 and $13 \mathrm{TeV}$ runs of the LHC as well as present several projections in future luminosities.

the ratio $c_{B} / c_{W}$, which controls the relative size of the branching ratios. ${ }^{4}$ As a consequence, the excluded region for each signature has the shape of a symmetric triangular angular slice in the $\left(c_{B}, c_{W}\right)$ plane. The strongest constraints come from the $Z Z$ and $Z \gamma$ final states. The $W W$ limit instead provides inferior exclusion power for regions already bounded by the other searches. The white region is compatible with all existing LHC $8 \mathrm{TeV}$ constraints and fits the $13 \mathrm{TeV}$ diphoton excess.

The remaining panels of figure 1 show the $\mathrm{LHC} 13 \mathrm{TeV}$ reach with increasing luminosity up to $3000 \mathrm{fb}^{-1}$. It is interesting to observe that the $Z \gamma$ limit at $3.2 \mathrm{fb}^{-1}$ is essentially equivalent to the $8 \mathrm{TeV}$ bound, while the $W W$ and the $Z Z$ are slightly weaker. Increasing the luminosity reduces the allowed parameter regions, resulting in a tiny remaining portion at $\mathcal{L}=3000 \mathrm{fb}^{-1}$. The result suggests that, if the diphoton excess is confirmed, a complementary signature in weak boson final states is highly likely to be discovered in the coming years. Notice that the projection of the $8 \mathrm{TeV}$ combined $Z Z$ limit we obtained in section 2 plays a crucial role in closing almost entirely the allowed parameter region at the high luminosity LHC.

\footnotetext{
${ }^{4}$ Note, however, that what we are imposing is a signal cross section in $\gamma \gamma$ at $13 \mathrm{TeV}$. In the transition from the gluon-fusion to the photon-fusion regime, the corresponding $8 \mathrm{TeV} \gamma \gamma$ signal strength changes since the $8 \mathrm{TeV} / 13 \mathrm{TeV}$ ratio of the gluon and photon luminosity is different. This effect is not visible in the shape of the regions excluded by the $8 \mathrm{TeV}$ searches since effectively they always lie inside the region dominated by gluon-fusion.
} 
In order to illustrate the effect of varying signal cross section on the LHC reach, we repeated the analysis from figure 1 for $\sigma_{\gamma \gamma}^{*}=3 \mathrm{fb}$. Figure 2 shows the results. Compared to results in figure 1 , the region where photon fusion cross section exceeds the required signal strength, extends to smaller values of $c_{W, B}$, reducing slightly the allowed parameter space which can accommodate the signal. Conversely, the $Z \gamma, W W$ and $Z Z$ limits are less stringent than in figure 1 , due to the smaller required signal cross section for the di-photon resonance. Note that the last panel of figure 2 suggests that it will be difficult to completely probe di-photon resonance models with signal strength of $\lesssim 3 \mathrm{fb}$, even with $3000 \mathrm{fb}^{-1}$ of integrated luminosity.

\subsection{The dark matter model: $g_{X} \neq 0, g_{f}=0$}

Current ATLAS results favor the interpretation of the diphoton excess in terms of a resonance with a relatively large width (i.e. $\Gamma / M \sim 5 \%$ ). The large width cannot be explained by decays to gluons and photons alone. Unitarity and the existing di-jet bounds exclude the coupling sizes necessary to generate the large width [40], suggesting that a wide $750 \mathrm{GeV}$ resonance would have to decay to other states as well. As no new charged particles with mass $\sim O(100 \mathrm{GeV})$ have been observed at the LHC, it is reasonable to consider that the large resonance width can be explained by decays to new invisible particles. Decays of the $750 \mathrm{GeV}$ resonance to neutral states are conceptually very interesting, as non-SM massive particles with no electric charge are natural candidates for dark matter.

Reference [7-10] already considered scenarios in which a scalar $S$ with mass of $750 \mathrm{GeV}$ is allowed to decay to dark matter. A generic feature appears in most models which explain the large width of $S$ via decays to dark matter: once the values for the decay width and dark matter relic density are fixed, the parameters of the dark sector $\left(m_{X}, g_{X}\right)$ are fully determined. For instance, in cases where dark matter is a Dirac fermion coupling to a pure scalar $S$, a large $S$ width and dark matter relic density predict $m_{X} \approx 300 \mathrm{GeV}, g_{X} \approx 2 .{ }^{5}$

As an illustration of the LHC prospects to probe the class of the dark matter models for the $750 \mathrm{GeV}$ resonance, here we will consider a benchmark point from ref. [7] which is allowed by the current astro-physical and collider constraints:

$$
m_{X}=320 \mathrm{GeV}, \quad g_{X}=2.6 .
$$

The first panel of figure 3 shows in solid red contours the $c_{G}$ values necessary to explain the diphoton excess in the dark matter model. The required values of $c_{G}$ at a fixed $\left(c_{B}, c_{W}\right)$ are significantly higher compared to the vanilla scenario of the previous section. The reason for a larger $c_{G}$ stems from the fact that in our dark matter model $\operatorname{Br}(S \rightarrow X \bar{X}) \approx 1$, requiring larger $c_{G}$ couplings to compensate for a smaller $\operatorname{Br}(S \rightarrow g g)$. Notice also that the photon fusion contribution to the $S$ production becomes dominant only for $c_{B}, c_{W} \gtrsim 2$.

The remaining panels of figure 3 show the results of the current LHC exclusion of the dark matter model parameter space as well as the future prospects. The main difference

\footnotetext{
${ }^{5}$ The values of the fixed parameter point can change based on the assumptions on the spin and CP properties of dark matter and $S$.
} 

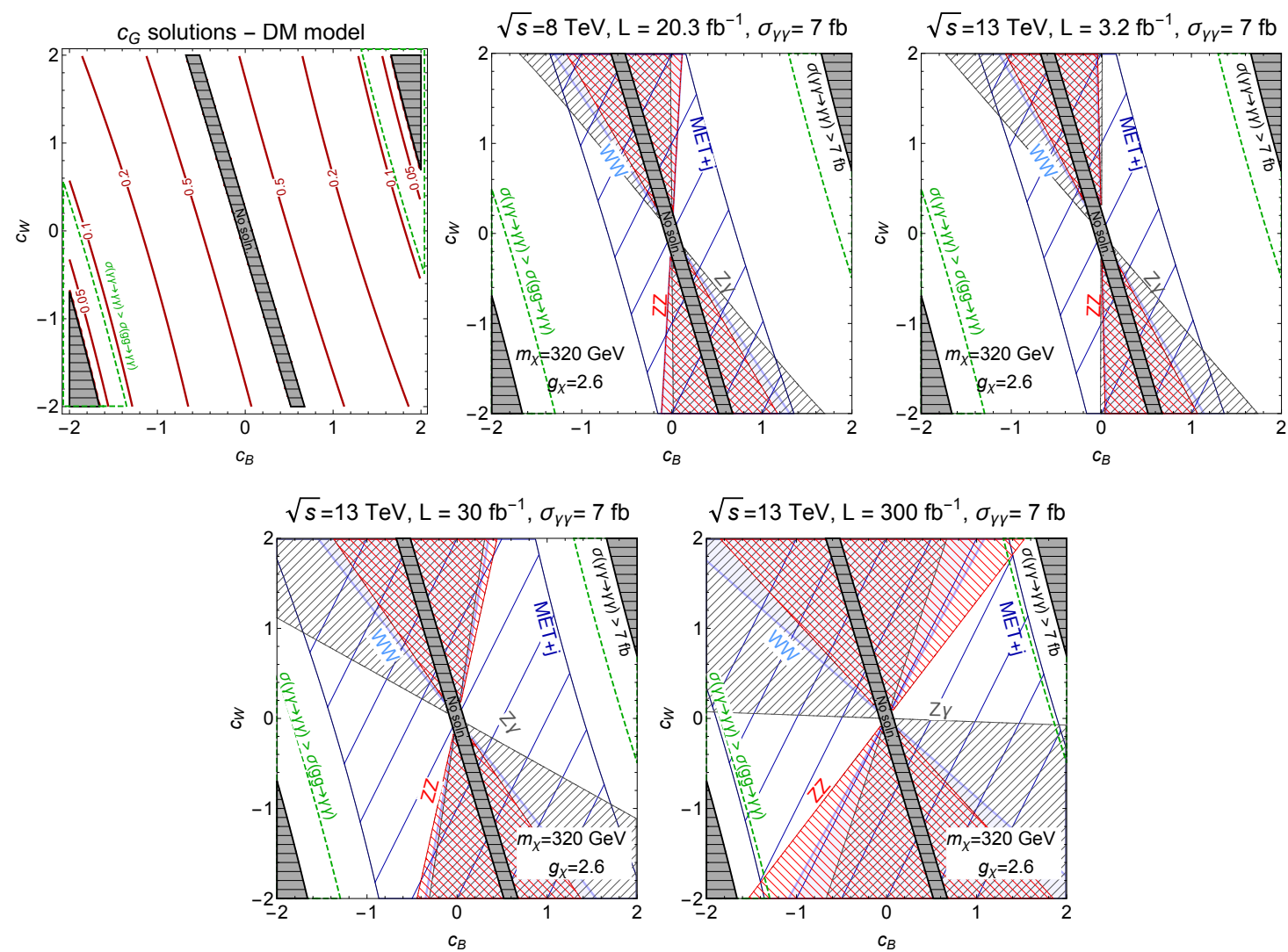

Figure 3. Analysis of the $\left(c_{B}, c_{W}\right)$ plane in the dark matter model parameter space. We apply the bounds from the 8 and $13 \mathrm{TeV}$ runs of the LHC as well as present several projections of bounds at future luminosities. The dark gray shaded regions are where there is no solution for $c_{G}$ which can accommodate the diphoton excess. Other shaded regions are excluded by particular searches labeled on the plots.

compared to the vanilla benchmark model of the previous section is that allowing the $750 \mathrm{GeV}$ scalar decays to dark matter introduces constraints from searches in channels with large missing energy, of which we consider MET $+j$. The $W W, Z Z$, and $\gamma Z$ results constrain the same regions of the parameter space as in the case of the vanilla model, while the MET $+j$ channel typically provides the strongest limits, except in the corners of large $\left(-c_{B}, c_{W}\right)$. We find that current $8 \mathrm{TeV}$ and $13 \mathrm{TeV}$ results exclude $c_{B}$ values in the range of $\left|c_{B}\right| \lesssim 0.2$ for $c_{W}=2$, up to values of $\left|c_{B}\right| \lesssim 1.7$ for $c_{W}=-2$. Future LHC results at $13 \mathrm{TeV}$ will be able to exclude a majority of the parameter space with as little as $30 \mathrm{fb}^{-1}$ of data, while with $300 \mathrm{fb}^{-1}$ only the regions of parameter space in which photon-fusion dominates will not be ruled out by $\mathrm{MET}+j$.

\subsection{The "top-philic" model: $g_{X}=0, g_{f} \neq 0$}

As a final concrete example of the diphoton models, we discuss the case in which the new scalar resonance also couples to SM fermions, i.e. we set $g_{X}=0$, with non-vanishing $g_{f}$ in eq. $(3.1){ }^{6}$

\footnotetext{
${ }^{6}$ Note that the coupling of $S$ to the SM fermions will generate extra contributions to the effective operator between $S$ and the gauge bosons (see for instance [41] for the case of a pseudoscalar coupled to
} 

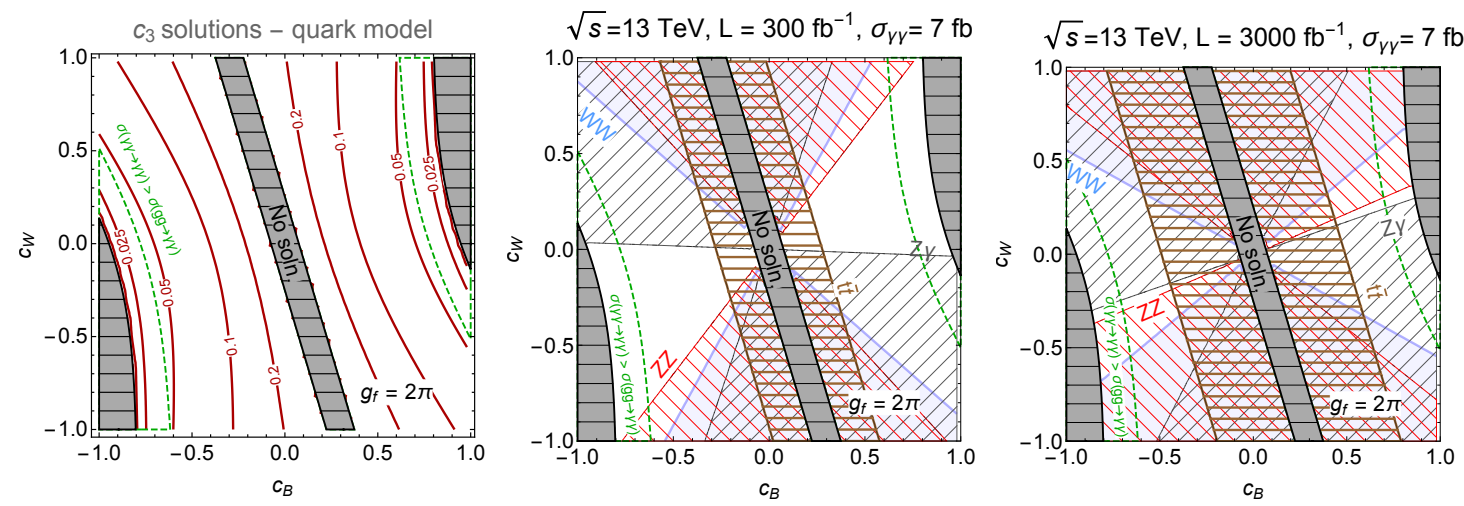

Figure 4. Analysis of the the top-philic model parameter space. The dark gray shaded regions are where there is no $c_{G}$ solution for the diphoton excess. Other shaded regions are excluded by particular searches labeled on the plots.

Among the various couplings to the SM fermions, the dominant coupling is to the top quark, justifying the title "top-philic". In particular, the coupling of $S$ with the top quark will induce a sizable decay width of $S$ into $\bar{t} t$ pairs (see appendix B), which now constitutes the dominant decay mode of the scalar resonance. As a consequence, in order to obtain the desired signal strength in the $\gamma \gamma$ final state at $13 \mathrm{TeV}$, the production cross section for $S$ should be quite sizeable compared to the vanilla model of section 3.1. The top-philic model is then similar to the dark matter model studied before, where the dominant invisible decay has been now substituted by a dominant decay into top-antitop pairs.

We show the results of our analysis in the case of the top-philic model in figure 4 for one representative value $g_{f}=2 \pi$ at the high luminosity LHC. Indeed, note that since the coupling to SM fermions are suppressed by a factor $m_{q} / \Lambda$ only large values of $g_{f}$ will induce interesting effects. The only final state which distinguishes the top-philic model from the vanilla scenario of section 3.1 is $t \bar{t}$. The brown shaded regions in figure 4 illustrate the regions of the parameter space the future $t \bar{t}$ resonance searches will be able to probe. The example we show in figure 4 suggests that the top-philic model can be probed with high luminosity LHC only in the regime of $g_{f} \gtrsim \pi$. In the large $g_{f}$ scenario, the addition of the $t \bar{t}$ channel to the usual electroweak boson searches essentially covers the entire parameter space that we considered with $3000 \mathrm{fb}^{-1}$ of integrated luminosity at $13 \mathrm{TeV}$ LHC. Note that the presence of a large coupling to the top quark, pushes the photon-fusion dominated region further to larger values of $c_{B}$ compared to the vanilla model of section 3.1. The reason is that a large coupling to $\mathrm{SM}$ fermions implies a small $\operatorname{Br}(S \rightarrow \gamma \gamma)$, resulting in the need of larger production cross section to accommodate the excess, that can essentially be obtained only via gluon fusion in the range of $c_{B}$ under consideration.

As a final remark, we note that we omitted the effects of $t \bar{t}$ interference with the SM model di-top production [42, 43]. The peak-dip structure in the $t \bar{t}$ invariant mass distribution resulting from interference is expected to be less pronounced for widths $\lesssim 1 \%$ of

gauge bosons and top quark). However, since we consider the same suppression scale $\Lambda$ for all dimension five operators, and all couplings $\left(c_{B}, c_{W}, c_{G}\right)$ and $g_{f}$ of order $O(1)$, such loop induced contributions will be typically subleading on the parameter space under study. 
the resonance mass. In our top-philic benchmark scenario, even taking $g_{f}=2 \pi$ results in a width which is $\sim O(1) \mathrm{GeV}$, suggesting that the interference effects can safely be neglected. Furthermore, the actual size of the interference term is model dependent, implying that even if the width is large, our result on the sensitivity of the $t \bar{t}$ searches can always be considered as the optimistic scenario.

\section{Summary}

In this paper we have explored the $\mathrm{LHC} 13 \mathrm{TeV}$ reach for models capable of explaining the diphoton excess at $750 \mathrm{GeV}$. As illustrative example we have considered a simple model with a scalar resonance coupled to SM gauge bosons, a dark matter candidate, and the SM quarks. We took into account gluon-fusion as well as photon-fusion as production mechanisms at the LHC. The requirement of generating the correct cross section in $\gamma \gamma$ final state at $13 \mathrm{TeV}$ imposes relations among the model parameters. We have studied the correlated signatures that can arise in such scenarios, including final states with dibosons, jet plus missing energy, and $t \bar{t}$ resonance searches, in order to further constrain the parameter space of the model and establish the exclusion reach of the LHC $13 \mathrm{TeV}$.

Our findings indicate that correlated LHC searches can exclude most of the relevant parameter space of a broad class of diphoton models during the second run of the LHC. The "vanilla" model (where the scalar resonance is coupled only to SM gauge bosons with dimension five operators) can be almost completely covered by associated signals in dibosons with $3000 \mathrm{fb}^{-1}$ of integrated luminosity. Concerning models where $S$ is a portal to a dark sector, we show that the mono-jet searches are able to corner the model with as little as $30 \mathrm{fb}^{-1}$. Finally, for models where the scalar resonance couples to SM quarks, the signature in the $t \bar{t}$ final state could provide a handle on distinguishing such scenarios from the "vanilla" model. However, in order for the signal in $t \bar{t}$ to be accessible, sizeable couplings of quarks to $S$ are required, as well as integrated luminosity of at least $300 \mathrm{fb}^{-1}$.

It would be interesting to extend our work to more exotic scenarios that can explain the diphoton excess, including e.g. non-resonant production, collimated photons, and models with non trivial coupling with the Higgs boson. The procedure we have adopted in this paper to compare with extrapolated LHC $13 \mathrm{TeV}$ limits could be extended also to such scenarios. If the di-photon excess is confirmed, it becomes of utmost importance to explore the full set of correlated signatures expected to appear in the ongoing run of the LHC.

Note added: during the final stages of this work, refs. [44] and [45] appeared. Both references studied the LHC prospects for exclusion of a simplified diphoton resonance model analogous to the scenario we study in section 3.1, and obtained results which are in agreement with ours. Compared to refs. [44] and [45], our analysis in section 3.1 also discusses the production mechanism for the resonance, including gluon and photon fusion. In addition, for the limits on the $Z Z$ production cross section we employ a $13 \mathrm{TeV}$ extrapolation of the the combined $8 \mathrm{TeV}$ limit, while ref. [44] discusses the current $13 \mathrm{TeV}$ limit on $Z Z$ production from the $l l \nu \nu$ final state only. 


\section{Acknowledgments}

We would like to thank A. Goudelis, K. Kowalska, D. Redigolo, F. Maltoni, E. Vryonidou, D. Pagani and F. Sala for useful discussions. M.B. is supported by a MOVE-IN Louvain Cofund grant. S.K. is supported by the "New Frontiers" program of the Austrian Academy of Sciences. A.M. is supported by the Strategic Research Program High Energy Physics and the Research Council of the Vrije Universiteit Brussel. M.B. and A.M. are also supported in part by the Belgian Federal Science Policy Office through the Interuniversity Attraction Pole P7/37. M.S. is supported in part by the European Commission through the "HiggsTools" Initial Training Network PITN-GA-2012-316704.

\section{A Limit extrapolation}

In order to project the $\mathrm{LHC} 8 \mathrm{TeV}$ limits to $13 \mathrm{TeV}$, we employ a simple extrapolation algorithm, similar to refs. $[46,47]$. We begin with the assumption that the $8 \mathrm{TeV}$ and $13 \mathrm{TeV}$ resonance searches are characterized by acceptances and event selection efficiencies which are roughly equal.

The $C L_{s}$ test statistics employed by the experimental collaborations to determine the 95\% C.L. upper bounds on the cross section times branching ratio is a constant at different luminosities and center of mass energies:

$$
C L_{s}(s, \mathcal{L}, M) \equiv C L_{s}\left(S^{\max }(s, \mathcal{L}, M), B(s, \mathcal{L}, M)\right)=95 \%=\text { const },
$$

where $S^{\max }$ is the upper bound on the number of signal events and $B$ is the expected or observed background, $s$ is the center of mass energy, $\mathcal{L}$ the integrated luminosity, and $M$ the invariant mass bin.

Assuming that the background is dominated by a single initial state production mode (which is a decent approximation in most cases) we can write at any given $\mathcal{L}$ and $M$ :

$$
B(s, \mathcal{L}, M)=r_{i j}(M, s) \times B\left(s^{0}, \mathcal{L}, M\right),
$$

where $r_{i j}$ is the parton luminosity ratio and $i, j$ stand for quarks and gluons.

Inserting this in eq. (A.1)

$$
\begin{aligned}
C L_{s}\left(S^{\max }(s, \mathcal{L}, M), B(s, \mathcal{L}, M)\right) & =C L_{s}\left(S^{\max }(s, \mathcal{L}, M), r_{i j}(M, s) \times B\left(s^{0}, \mathcal{L}, M\right)\right) \\
& =C L_{s}\left(S^{\max }(s, \mathcal{L}, M), r_{i j}(M, s) \times \frac{\mathcal{L}}{\mathcal{L}^{0}} B\left(s^{0}, \mathcal{L}^{0}, M\right)\right) \\
& =C L_{s}\left(S^{\max }\left(s^{0}, \mathcal{L}^{0}, M\right), B\left(s^{0}, \mathcal{L}^{0}, M\right)\right)
\end{aligned}
$$

where in the last line we have used the fact the the $C L_{s}$ is a constant, see eq. (A.1).

In the limit of a large number of events the event ditribution becomes well approximated by a Gaussian, so that the equality between the second-to-last and last line of eq. (A.3) can be written as

$$
\frac{S^{\max }(s, \mathcal{L}, M)}{\left[r_{i j}(M, s) \times \frac{\mathcal{L}}{\mathcal{L}^{0}} B\left(s^{0}, \mathcal{L}^{0}, M\right)\right]^{1 / 2}}=\frac{S^{\max }\left(s^{0}, \mathcal{L}^{0}, M\right)}{B\left(s^{0}, \mathcal{L}^{0}, M\right)^{1 / 2}} .
$$


Moreover, because of our initial assumption that the efficiencies and acceptances are the same, $S^{\max }(s, \mathcal{L}, M)$ scales as $\mathcal{L} \times \sigma^{\max }(s, M)$, so that one solves eq. (A.4) to get

$$
\sigma^{\max }(s, M) \approx \sqrt{r_{i j}(M, s)} \times \sqrt{\frac{\mathcal{L}^{0}}{\mathcal{L}}} \times \sigma^{\max }\left(s^{0}, M\right) .
$$

Equation (A.5) represents our "master formula" for limit extrapolations. The parton luminosity ratios $r_{i j}(M, s)$ have been previously calculated in ref. [48]. For completeness, here we give a numerical polynomial fit to the parton luminosity ratios for $g g, \sum(q q+q \bar{q})$ and $q g$ initial states, valid in the range of $M=50-4000 \mathrm{GeV}$ :

$$
\begin{aligned}
r_{g g}(x) \approx & 1.6+6.3 \times 10^{-3} x-7.9 \times 10^{-6} x^{2}+8.8 \times 10^{-9} x^{3} \\
& -3.7 \times 10^{-12} x^{4}+6.6 \times 10^{-16} x^{5}, \\
r_{q q}(x) \approx & 1.7-2.5 x+6.0 \times 10^{-6} x^{2}-8.8 \times 10^{-9} x^{3} \\
& +6.2 \times 10^{-12} x^{4}-1.9 \times 10^{-15} x^{5}+2.3 \times 10^{-19} x^{6}, \\
r_{q g}(x) \approx & 1.3+7.1 \times 10^{-3} x-1.2 \times 10^{-5} x^{2} \\
& +1.1 \times 10^{-8} x^{3}-4.1 \times 10^{-12} x^{4}+5.7 \times 10^{-16} x^{5},
\end{aligned}
$$

where $x \equiv M / \mathrm{GeV}$.

We find that when used to extrapolate the expected $8 \mathrm{TeV}$ limits, the extrapolation formula of eq. (A.5) gives results which are within $\sim 20 \%$ from the true expected limits at $13 \mathrm{TeV}$. In order to validate the procedure, we have compared the results using eq. (A.5) to a number of already public ATLAS results from $13 \mathrm{TeV}$. Table 2 shows the results. The largest error in our limit extrapolation is $\sim 28-29 \%$, in the case of the $\gamma \gamma$ and $Z \gamma$ searches. This is mostly due to the fact that ATLAS does not provide for those searches the efficiencies for all bins, and a knowledge of the latter is required to extrapolate the cross section bound for the fiducial cross section bound. The average error is about $10 \%$. The uncertainty in the limit extrapolation does not strongly affect our results on the parameter space exclusion. Figure 5 illustrates the result in case of the $W W$ cross section, extrapolated from the $8 \mathrm{TeV}$ limit to $13 \mathrm{TeV}$ with $\mathcal{L}=3000 \mathrm{fb}^{-1}$. The blue, shaded region shows the excluded parameter space, while the dashed regions show where the edge of the exclusion would lie if the maximal cross section was $\pm 20 \%$ different.

Although eq. (A.5) gives reasonably accurate results in many cases, it is important to point out where it fails. If the event reconstruction and selection efficiencies and acceptances differ significantly between $8 \mathrm{TeV}$ and $13 \mathrm{TeV}$, eq. (A.5) can result in errors larger than $20 \%$. The approximation is also not accurate when the $8 \mathrm{TeV}$ expected background is a number of the order of a few units, so that the event distribution is not well approximated by a Gaussian, but rather presents a longer tail.

Another scenario in which the extrapolation of eq. (A.5) fails are non-resonance searches (e.g. MET $+j$ ) or searches for broad resonances. In cases where the signal cross section is not distributed mostly in a narrow range of invariant masses (such as in the case of a narrow resonance), it is inappropriate to use a parton luminosity ratio evaluated at a single $M$. Instead, an integral value over the parton luminosities is more appropriate, as the signal cross section will be distributed over a wider range of $M$. 


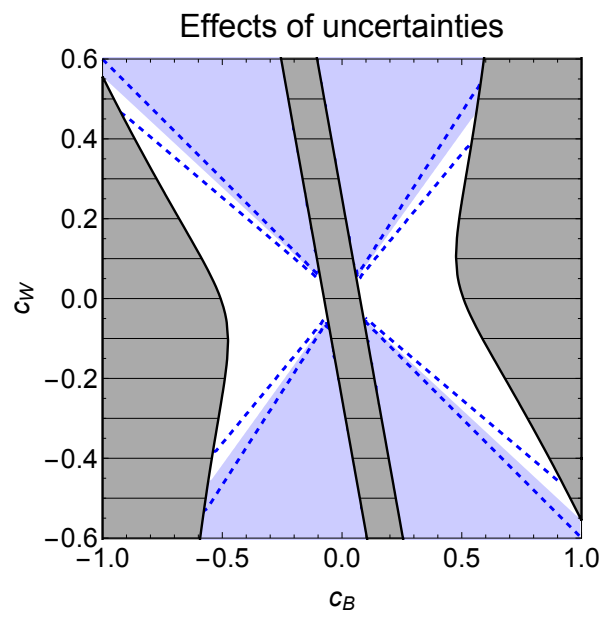

Figure 5. Effects of uncertainties of the limit extrapolation procedure on the exclusion regions of the model parameter space. The blue, shaded region shows the portion of the "base model" parameter space excluded by the extrapolated $W W$ limit at $13 \mathrm{TeV}$ with $\mathcal{L}=3000 \mathrm{fb}^{-1}$, with the dashed lines showing the position of the excluded region edges if the limit on the cross section was $\pm 20 \%$ different. The gray shaded regions represent the parameter space where either no-viable solution for $c_{G}$ can be found to accommodate the excess or the predicted diphoton cross section at $13 \mathrm{TeV}$ is too big.

\begin{tabular}{|c|c|c|c|c|c|c|c|}
\hline F.S. & Ref. & $M_{\mathrm{res}}[\mathrm{GeV}]$ & I.S. & $\sigma_{\exp }^{\max }, \mathcal{L}(8 \mathrm{TeV})$ & $\sigma_{\exp }^{\max }, \mathcal{L}(13 \mathrm{TeV})$ & $\sigma_{\text {ext }}^{\max }(13 \mathrm{TeV})$ & $\%$ diff. \\
\hline \multirow{4}{*}{$Z h$} & \multirow{2}{*}{$8 \mathrm{TeV}[49]$} & 300 & \multirow{4}{*}{$g g$} & $220 \mathrm{fb}, 20.3 \mathrm{fb}^{-1}$ & $1250 \mathrm{fb}, 3.2 \mathrm{fb}^{-1}$ & $952 \mathrm{fb}$ & $-27 \%$ \\
\hline & & 400 & & $92 \mathrm{fb}, 20.3 \mathrm{fb}^{-1}$ & $500 \mathrm{fb}, 3.2 \mathrm{fb}^{-1}$ & $423 \mathrm{fb}$ & $-17 \%$ \\
\hline & \multirow{2}{*}{$13 \mathrm{TeV}[50]$} & 750 & & $16 \mathrm{fb}, 20.3 \mathrm{fb}^{-1}$ & $73 \mathrm{fb}, 3.2 \mathrm{fb}^{-1}$ & $87 \mathrm{fb}$ & $+17 \%$ \\
\hline & & 1000 & & $10 \mathrm{fb}, 20.3 \mathrm{fb}^{-1}$ & $50 \mathrm{fb}, 3.2 \mathrm{fb}^{-1}$ & $61 \mathrm{fb}$ & $+20 \%$ \\
\hline \multirow{3}{*}{$Z_{\gamma}$} & $8 \mathrm{ToV}[22]$ & 400 & \multirow{3}{*}{$q q$} & $0.5 \mathrm{fb}, 20.3 \mathrm{fb}^{-1}$ & $2.3 \mathrm{fb}, 3.2 \mathrm{fb}^{-1}$ & $1.8 \mathrm{fb}$ & $-24 \%$ \\
\hline & $81 \mathrm{ev}[23]$ & 750 & & $0.2 \mathrm{fb}, 20.3 \mathrm{fb}^{-1}$ & $1.2 \mathrm{fb}, 3.2 \mathrm{fb}^{-1}$ & $0.9 \mathrm{fb}$ & $-29 \%$ \\
\hline & $13 \mathrm{TeV}$ [24] & 1600 & & $0.1 \mathrm{fb}, 20.3 \mathrm{fb}^{-1}$ & $0.6 \mathrm{fb}, 3.2 \mathrm{fb}^{-1}$ & $0.6 \mathrm{fb}$ & $0 \%$ \\
\hline \multirow{3}{*}{$l l$} & $8 \mathrm{ToV}[51]$ & 500 & \multirow{3}{*}{$q q$} & $3.2 \mathrm{fb}, 20.4 \mathrm{fb}^{-1}$ & $11 \mathrm{fb}, 3.2 \mathrm{fb}^{-1}$ & $12 \mathrm{fb}$ & $+9 \%$ \\
\hline & $81 \mathrm{ev}[51]$ & 750 & & $1.2 \mathrm{fb}, 20.4 \mathrm{fb}^{-1}$ & $4.8 \mathrm{fb}, 3.2 \mathrm{fb}^{-1}$ & $4.9 \mathrm{fb}$ & $+2 \%$ \\
\hline & $13 \mathrm{TeV}[52]$ & 1500 & & $0.4 \mathrm{fb}, 20.4 \mathrm{fb}^{-1}$ & $1.6 \mathrm{fb}, 3.2 \mathrm{fb}^{-1}$ & $1.9 \mathrm{fb}$ & $+17 \%$ \\
\hline \multirow{3}{*}{$\begin{array}{c}Z Z \\
(l l q q)\end{array}$} & & 750 & \multirow{3}{*}{$q q$} & $48 \mathrm{fb}, 20.3 \mathrm{fb}^{-1}$ & $200 \mathrm{fb}, 3.2 \mathrm{fb}^{-1}$ & $197 \mathrm{fb}$ & $-2 \%$ \\
\hline & & 1000 & & $19 \mathrm{fb}, 20.3 \mathrm{fb}^{-1}$ & $105 \mathrm{fb}, 3.2 \mathrm{fb}^{-1}$ & $85 \mathrm{fb}$ & $-21 \%$ \\
\hline & $13 \mathrm{TeV}[36]$ & 2000 & & $6.0 \mathrm{fb}, 20.3 \mathrm{fb}^{-1}$ & $38 \mathrm{fb}, 3.2 \mathrm{fb}^{-1}$ & $41 \mathrm{fb}$ & $+8 \%$ \\
\hline \multirow{3}{*}{$h h$} & $8 \mathrm{ToV}[54]$ & 600 & \multirow{3}{*}{$g g$} & $22 \mathrm{fb}, 19.5 \mathrm{fb}^{-1}$ & $110 \mathrm{fb}, 3.2 \mathrm{fb}^{-1}$ & $110 \mathrm{fb}$ & $0 \%$ \\
\hline & $81 \mathrm{e}$ [54] & 800 & & $9 \mathrm{fb}, 19.5 \mathrm{fb}^{-1}$ & $60 \mathrm{fb}, 3.2 \mathrm{fb}^{-1}$ & $49 \mathrm{fb}$ & $-20 \%$ \\
\hline & $13 \mathrm{TeV}$ [55] & 1400 & & $3.9 \mathrm{fb}, 19.5 \mathrm{fb}^{-1}$ & $22 \mathrm{fb}, 3.2 \mathrm{fb}^{-1}$ & $28 \mathrm{fb}$ & $+24 \%$ \\
\hline \multirow{3}{*}{$\gamma \gamma$} & 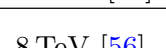 & 500 & \multirow{3}{*}{$g g$} & $4.1 \mathrm{fb}, 20.3 \mathrm{fb}^{-1}$ & $22 \mathrm{fb}, 3.2 \mathrm{fb}^{-1}$ & $20 \mathrm{fb}$ & $-10 \%$ \\
\hline & $81 \mathrm{e}[56]$ & 750 & & $2.0 \mathrm{fb}, 20.3 \mathrm{fb}^{-1}$ & $8.2 \mathrm{fb}, 3.2 \mathrm{fb}^{-1}$ & $10.9 \mathrm{fb}$ & $+28 \%$ \\
\hline & $13 \mathrm{TeV}[1]$ & 1500 & & $0.5 \mathrm{fb}, 20.3 \mathrm{fb}^{-1}$ & $3.9 \mathrm{fb}, 3.2 \mathrm{fb}^{-1}$ & $3.8 \mathrm{fb}$ & $-3 \%$ \\
\hline
\end{tabular}

Table 2. Validations of the limit extrapolation procedure from 8 to $13 \mathrm{TeV}$. In the table F.S. stands for decay "final state" and I.S. for production "initial state". We extracted the expected limits from the corresponding references listed in the table. Percent difference is defined as $2\left[\sigma_{\text {ext }}^{\max }(13 \mathrm{TeV})-\sigma_{\exp }^{\max }(13 \mathrm{TeV})\right] /\left[\sigma_{\text {ext }}^{\max }(13 \mathrm{TeV})+\sigma_{\exp }^{\max }(13 \mathrm{TeV})\right]$. Extrapolations are accurate within a $\sim 20 \%$ margin. The only shown exception involves the $750 \mathrm{GeV}$ bin of the $\gamma \gamma$ search, as [1] does not provide a detailed account of the acceptances/efficiencies in all bins, which are necessary when comparing the "fiducial' cross section to the physical cross section. 


\section{B Analytical form of the decay widths}

In this appendix we report the analytic formulas for the partial decay widths of the resonance. The following expressions were used for the analysis discussed in the main body of the paper.

$$
\begin{aligned}
\Gamma[S \rightarrow \gamma \gamma] & =\frac{\left(c_{B} \cos ^{2} \theta_{W}+c_{W} \sin ^{2} \theta_{W}\right)^{2} m_{S}^{3}}{4 \pi \Lambda^{2}} \\
\Gamma[S \rightarrow g g] & =\frac{2 c_{G}^{2} m_{S}^{3}}{\Lambda^{2} \pi} \\
\Gamma[S \rightarrow Z Z] & =\frac{\left(c_{B} \sin ^{2} \theta_{W}+c_{W} \cos ^{2} \theta_{W}\right)^{2} m_{S}^{3}}{4 \pi \Lambda^{2}}\left(1-4 \frac{m_{Z}^{2}}{m_{S}^{2}}+6 \frac{m_{Z}^{4}}{m_{S}^{4}}\right) \sqrt{1-\frac{4 m_{Z}^{2}}{m_{S}^{2}}} \\
\Gamma[S \rightarrow Z \gamma] & =\frac{\left(\sin \theta_{W} \cos \theta_{W}\left(c_{B}-c_{W}\right)\right)^{2} m_{S}^{3}}{2 \pi \Lambda^{2}}\left(1-\frac{m_{Z}^{2}}{m_{S}}\right)^{3} \\
\Gamma\left[S \rightarrow W^{+} W^{-}\right] & =\frac{c_{W}^{2} m_{S}^{3}}{2 \pi \Lambda^{2}}\left(1-4 \frac{m_{W}^{2}}{m_{S}^{2}}+6 \frac{m_{W}^{4}}{m_{S}^{4}}\right) \sqrt{1-\frac{4 m_{W}^{2}}{m_{S}^{2}}} \\
\Gamma[S \rightarrow \bar{X} X] & =\frac{g_{X}^{2} m_{S}}{8 \pi}\left(1-\frac{4 m_{X}^{2}}{m_{S}^{2}}\right)^{3 / 2} \\
\Gamma[S \rightarrow \bar{q} q] & =\frac{3 g_{f}^{2} m_{S} m_{q}^{2}}{8 \pi \Lambda^{2}}\left(1-\frac{4 m_{q}^{2}}{m_{S}^{2}}\right)^{3 / 2}
\end{aligned}
$$

Open Access. This article is distributed under the terms of the Creative Commons Attribution License (CC-BY 4.0), which permits any use, distribution and reproduction in any medium, provided the original author(s) and source are credited.

\section{References}

[1] ATLAS collaboration, Search for resonances decaying to photon pairs in $3.2 \mathrm{fb}^{-1}$ of $p p$ collisions at $\sqrt{s}=13 \mathrm{TeV}$ with the ATLAS detector, ATLAS-CONF-2015-081 (2015).

[2] ATLAS collaboration, Search for resonances in diphoton events with the ATLAS detector at $\sqrt{s}=13 \mathrm{TeV}$, ATLAS-CONF-2016-018 (2016).

[3] CMS collaboration, Search for new physics in high mass diphoton events in proton-proton collisions at $\sqrt{s}=13 \mathrm{TeV}$, CMS-PAS-EXO-15-004 (2015).

[4] CMS collaboration, Search for new physics in high mass diphoton events in $3.3 \mathrm{fb}^{-1}$ of proton-proton collisions at $\sqrt{s}=13 \mathrm{TeV}$ and combined interpretation of searches at $8 \mathrm{TeV}$ and $13 \mathrm{TeV}$, CMS-PAS-EXO-16-018 (2016).

[5] I. Low and J. Lykken, Implications of gauge invariance on a heavy diphoton resonance, arXiv:1512.09089 [INSPIRE].

[6] J.F. Kamenik, B.R. Safdi, Y. Soreq and J. Zupan, Comments on the diphoton excess: critical reappraisal of effective field theory interpretations, JHEP 07 (2016) 042 [arXiv: 1603. 06566] [INSPIRE]. 
[7] M. Backovic, A. Mariotti and D. Redigolo, Di-photon excess illuminates dark matter, JHEP 03 (2016) 157 [arXiv: 1512.04917] [INSPIRE].

[8] D. Barducci, A. Goudelis, S. Kulkarni and D. Sengupta, One jet to rule them all: monojet constraints and invisible decays of a $750 \mathrm{GeV}$ diphoton resonance, JHEP 05 (2016) 154 [arXiv: 1512.06842] [INSPIRE].

[9] Y. Mambrini, G. Arcadi and A. Djouadi, The LHC diphoton resonance and dark matter, Phys. Lett. B 755 (2016) 426 [arXiv:1512.04913] [INSPIRE].

[10] F. D'Eramo, J. de Vries and P. Panci, A $750 \mathrm{GeV}$ portal: LHC phenomenology and dark matter candidates, JHEP 05 (2016) 089 [arXiv:1601.01571] [INSPIRE].

[11] X.-J. Bi, Q.-F. Xiang, P.-F. Yin and Z.-H. Yu, The $750 \mathrm{GeV}$ diphoton excess at the LHC and dark matter constraints, Nucl. Phys. B 909 (2016) 43 [arXiv:1512.06787] [INSPIRE].

[12] R. Franceschini et al., What is the $\gamma \gamma$ resonance at $750 \mathrm{GeV}$ ?, JHEP 03 (2016) 144 [arXiv: 1512.04933] [INSPIRE].

[13] A. Falkowski, O. Slone and T. Volansky, Phenomenology of a $750 \mathrm{GeV}$ singlet, JHEP 02 (2016) 152 [arXiv : 1512.05777] [INSPIRE].

[14] L. Berthier, J.M. Cline, W. Shepherd and M. Trott, Effective interpretations of a diphoton excess, JHEP 04 (2016) 084 [arXiv: 1512.06799] [INSPIRE].

[15] R.S. Gupta, S. Jäger, Y. Kats, G. Perez and E. Stamou, Interpreting a $750 \mathrm{GeV}$ diphoton resonance, arXiv:1512.05332 [INSPIRE].

[16] C. Csáki, J. Hubisz and J. Terning, Minimal model of a diphoton resonance: production without gluon couplings, Phys. Rev. D 93 (2016) 035002 [arXiv:1512.05776] [INSPIRE].

[17] C. Csáki, J. Hubisz, S. Lombardo and J. Terning, Gluon versus photon production of a $750 \mathrm{GeV}$ diphoton resonance, Phys. Rev. D 93 (2016) 095020 [arXiv:1601.00638] [INSPIRE].

[18] L.A. Harland-Lang, V.A. Khoze and M.G. Ryskin, The production of a diphoton resonance via photon-photon fusion, JHEP 03 (2016) 182 [arXiv: 1601.07187] [INSPIRE].

[19] S. Abel and V.V. Khoze, Photo-production of a $750 \mathrm{GeV}$ di-photon resonance mediated by Kaluza-Klein leptons in the loop, JHEP 05 (2016) 063 [arXiv:1601.07167] [INSPIRE].

[20] S. Fichet, G. von Gersdorff and C. Royon, Scattering light by light at $750 \mathrm{GeV}$ at the LHC, Phys. Rev. D 93 (2016) 075031 [arXiv:1512.05751] [inSPIRE].

[21] J. Jaeckel, M. Jankowiak and M. Spannowsky, LHC probes the hidden sector, Phys. Dark Univ. 2 (2013) 111 [arXiv: 1212.3620] [INSPIRE].

[22] J. Alwall et al., Comparative study of various algorithms for the merging of parton showers and matrix elements in hadronic collisions, Eur. Phys. J. C 53 (2008) 473 [arXiv: 0706.2569] [INSPIRE].

[23] ATLAS collaboration, Search for new resonances in $W \gamma$ and $Z \gamma$ final states in pp collisions at $\sqrt{s}=8$ TeV with the ATLAS detector, Phys. Lett. B 738 (2014) 428 [arXiv:1407.8150] [INSPIRE].

[24] ATLAS collaboration, Search for heavy resonances decaying to a $Z$ boson and a photon in $p p$ collisions at $\sqrt{s}=13 \mathrm{TeV}$ with the ATLAS detector, ATLAS-CONF-2016-010 (2016).

[25] ATLAS collaboration, G. Aad et al., Search for an additional, heavy Higgs boson in the $H \rightarrow Z Z$ decay channel at $\sqrt{s}=8 \mathrm{TeV}$ in pp collision data with the ATLAS detector, Eur. Phys. J. C 76 (2016) 45 [arXiv:1507.05930]. 
[26] ATLAS collaboration, Search for high-mass resonances decaying into a $Z$ boson pair in the $\ell \ell \nu \nu$ final state in pp collisions at $\sqrt{s}=13 \mathrm{TeV}$ with the ATLAS detector,

ATLAS-CONF-2016-012 (2016).

[27] ATLAS collaboration, Search for a high-mass Higgs boson decaying to a $W$ boson pair in pp collisions at $\sqrt{s}=8 \mathrm{TeV}$ with the ATLAS detector, JHEP 01 (2016) 032

[arXiv: 1509.00389] [INSPIRE].

[28] ATLAS collaboration, Search for a high-mass Higgs boson decaying to a pair of $W$ bosons in pp collisions at $\sqrt{s}=13 \mathrm{TeV}$ with the ATLAS detector, ATLAS-CONF-2016-021 (2016).

[29] CMS collaboration, Searches for new physics using the $t \bar{t}$ invariant mass distribution in pp collisions at $\sqrt{s}=8 \mathrm{TeV}$, Phys. Rev. Lett. 111 (2013) 211804 [arXiv:1309. 2030] [InSPIRE].

[30] ATLAS collaboration, Search for heavy particles decaying to pairs of highly-boosted top quarks using lepton-plus-jets events in proton-proton collisions at $\sqrt{s}=13 \mathrm{TeV}$ with the ATLAS detector, ATLAS-CONF-2016-014 (2016).

[31] ATLAS collaboration, Search for new phenomena in final states with an energetic jet and large missing transverse momentum in pp collisions at $\sqrt{s}=8 \mathrm{TeV}$ with the ATLAS detector, Eur. Phys. J. C 75 (2015) 299 [arXiv:1502.01518] [inSPIRE].

[32] ATLAS collaboration, Search for new phenomena in final states with an energetic jet and large missing transverse momentum in pp collisions at $\sqrt{s}=13 \mathrm{TeV}$ using the ATLAS detector, arXiv:1604.07773 [INSPIRE].

[33] A. Abdesselam et al., Boosted objects: a probe of beyond the standard model physics, Eur. Phys. J. C 71 (2011) 1661 [arXiv:1012.5412] [INSPIRE].

[34] A. Altheimer et al., Jet substructure at the Tevatron and LHC: new results, new tools, new benchmarks, J. Phys. G 39 (2012) 063001 [arXiv:1201.0008] [INSPIRE].

[35] ATLAS collaboration, Search for diboson resonances in the $\nu \nu q q$ final state in pp collisions at $\sqrt{s}=13 \mathrm{TeV}$ with the ATLAS detector, ATLAS-CONF-2015-068 (2015).

[36] ATLAS collaboration, Search for diboson resonances in the llqq final state in pp collisions at $\sqrt{s}=13 \mathrm{TeV}$ with the ATLAS detector, ATLAS-CONF-2015-071 (2015).

[37] A. Alloul, N.D. Christensen, C. Degrande, C. Duhr and B. Fuks, FeynRules 2.0 - A complete toolbox for tree-level phenomenology, Comput. Phys. Commun. 185 (2014) 2250 [arXiv: 1310.1921] [INSPIRE].

[38] J. Alwall et al., The automated computation of tree-level and next-to-leading order differential cross sections and their matching to parton shower simulations, JHEP 07 (2014) 079 [arXiv: 1405.0301] [INSPIRE].

[39] R.D. Ball et al., Parton distributions with LHC data, Nucl. Phys. B 867 (2013) 244 [arXiv: 1207.1303] [INSPIRE].

[40] S. Knapen, T. Melia, M. Papucci and K. Zurek, Rays of light from the LHC, Phys. Rev. D 93 (2016) 075020 [arXiv: 1512.04928] [INSPIRE].

[41] B. Bellazzini, R. Franceschini, F. Sala and J. Serra, Goldstones in diphotons, JHEP 04 (2016) 072 [arXiv: 1512.05330] [INSPIRE].

[42] A. Djouadi, J. Ellis and J. Quevillon, Interference effects in the decays of spin-zero resonances into $\gamma \gamma$ and $t \bar{t}, J H E P 07$ (2016) 105 [arXiv: 1605.00542] [INSPIRE]. 
[43] B. Hespel, F. Maltoni and E. Vryonidou, Signal background interference effects in heavy scalar production and decay to a top-anti-top pair, arXiv:1606.04149 [INSPIRE].

[44] R. Sato and K. Tobioka, LHC future prospects of the $750 \mathrm{GeV}$ resonance, arXiv:1605.05366 [INSPIRE].

[45] J.M. No, Is it $\mathrm{SU}(2)_{L}$ or just $\mathrm{U}(1)_{Y}$ ? $750 \mathrm{GeV}$ di-photon probes of the electroweak nature of new states, arXiv: 1605.05900 [INSPIRE].

[46] A. Thamm, R. Torre and A. Wulzer, Future tests of Higgs compositeness: direct vs. indirect, JHEP 07 (2015) 100 [arXiv: 1502.01701] [INSPIRE].

[47] D. Buttazzo, F. Sala and A. Tesi, Singlet-like Higgs bosons at present and future colliders, JHEP 11 (2015) 158 [arXiv:1505.05488] [INSPIRE].

[48] J.W. Sterling. personal communication.

[49] ATLAS collaboration, Search for a CP-odd Higgs boson decaying to Zh in pp collisions at $\sqrt{s}=8 \mathrm{TeV}$ with the ATLAS detector, Phys. Lett. B 744 (2015) 163 [arXiv:1502.04478] [INSPIRE].

[50] ATLAS collaboration, Search for a CP-odd Higgs boson decaying to Zh in pp collisions at $\sqrt{s}=13$ TeV with the ATLAS detector, ATLAS-CONF-2016-015 (2016).

[51] ATLAS collaboration, Search for high-mass dilepton resonances in pp collisions at $\sqrt{s}=8$ TeV with the ATLAS detector, Phys. Rev. D 90 (2014) 052005 [arXiv: 1405.4123] [InSPIRE].

[52] ATLAS collaboration, Search for new phenomena in the dilepton final state using proton-proton collisions at $\sqrt{s}=13$ TeV with the ATLAS detector, ATLAS-CONF-2015-070 (2015).

[53] ATLAS collaboration, Search for resonant diboson production in the $\ell \ell q \bar{q}$ final state in $p p$ collisions at $\sqrt{s}=8 \mathrm{TeV}$ with the ATLAS detector, Eur. Phys. J. C 75 (2015) 69 [arXiv: 1409.6190] [INSPIRE].

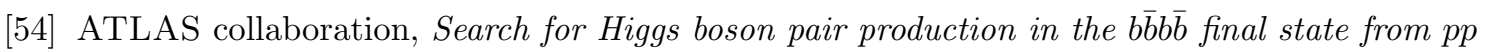
collisions at $\sqrt{s}=8 \mathrm{TeV}$ with the ATLAS detector, Eur. Phys. J. C 75 (2015) 412 [arXiv: 1506.00285] [INSPIRE].

[55] ATLAS collaboration, Search for pair production of Higgs bosons in the $b \bar{b} b \bar{b}$ final state using proton-proton collisions at $\sqrt{s}=13$ TeV with the ATLAS detector, ATLAS-CONF-2016-017 (2016).

[56] ATLAS collaboration, Search for high-mass diphoton resonances in pp collisions at $\sqrt{s}=8$ TeV with the ATLAS detector, Phys. Rev. D 92 (2015) 032004 [arXiv:1504.05511] [INSPIRE]. 